\title{
Research of kinematics of an actuating group of an anthropomorphic gripper with a common drive
}

\author{
Alexey Bogdanov ${ }^{1}$, Alexander Permyakov ${ }^{1}$, and Yulia Zhdanova ${ }^{2, *}$ \\ ${ }^{1}$ JSC 'SPA ‘Android technics', 23 Grayvoronovskaya st., Moscow 109518, Russia \\ ${ }^{2}$ Moscow Technological University (MIREA), 78 Vernadskij prosp., Moscow 119454, Russia
}

\begin{abstract}
Anthropomorphic robots (AR): Robonaut (USA), Asimo (Japan), FEDOR (Russia) are similar to human capabilities in performable actions. Whereby the AR efficiency depends on the anthropomorphic gripper (AG) functionality. The key problems while its developing are restrictions imposed on permissible amount of using motors. One of advanced approaches to developing of the AG with capabilities similar to a human hand is the use of a common drive wisawe3dsth a special motion transfer system (MTS). Due to including functioning connections into the MTS construction the variability of a schematic structure is provided and the kinematic dependence of output links motion is excluded, thus their sequence motion is provided. The inverse kinematic problem was solved for the developed MTS version. Analytical dependences joining the MTS parameters, rotational angles of actuating group links with a rotational angle of an output link drive are obtained. Performed researches are sufficient for the AG power analysis. Implementability of gripper links rotational angles similar to the phalanges rotation, when using the proposed MTS, is confirmed.
\end{abstract}

\section{Introduction}

One of rapidly developing technics areas are anthropomorphic robots (AR). It is caused by demand for technological complexes capable of functioning with capabilities similar to human and developing of software and hardware integral part of a control system.

Equipping AR with a complex similar to human basic sense organs allows their using for work in extreme conditions: on a space station [1 - 3], in high-threat areas [4].

Modern AR are capable of performing wide variety of active movements. AR "FEDOR" created by JSC "SPA "Android technics" (project "Lifesaver", funding by Advanced Research Foundation) in 2014 - 2016 is capable of moving with the speed up to $4 \mathrm{~km} / \mathrm{h}$, using power tool: drill, angle grinder, thrustor and so on. Realized technologies are based on the use of a modern element base and specific architecture of an actuating complex and control system. Developing anthropomorphic manipulators (AM) and an

\footnotetext{
* Corresponding author: musrosjk@gmail.com
} 
anthropomorphic gripper (AG) capable of interacting with external objects with different surface geometry provides performance of the greater part of 54 actions given in the terms of reference. AG is constructed in accordance with the technical solution patented in the Russian Federation [5] and comprises five actuating groups of links (AGL). Each AGL includes three output links. Total amount of AG kinematic couples is 15. In view of restrictions imposed on the size of the manipulator, a group drive of AGL links was used. It allowed reducing the amount of active AG drives to 6 (Fig. 1). The AG mass made 0,96 $\mathrm{kg}$. Motors, reducers and ballscrew were mounted on the manipulator link preceding the gripper. Two drives providing the gripper base movement are also disposed at the same place. Its mass makes $6,63 \mathrm{~kg}$. The possibilities of additional motors placement on the manipulator link are almost exhausted.

In view of the group drive use the AGL links motions are kinematically dependent. It reduces the range of captured details sizes with realization of a multiple links contact.

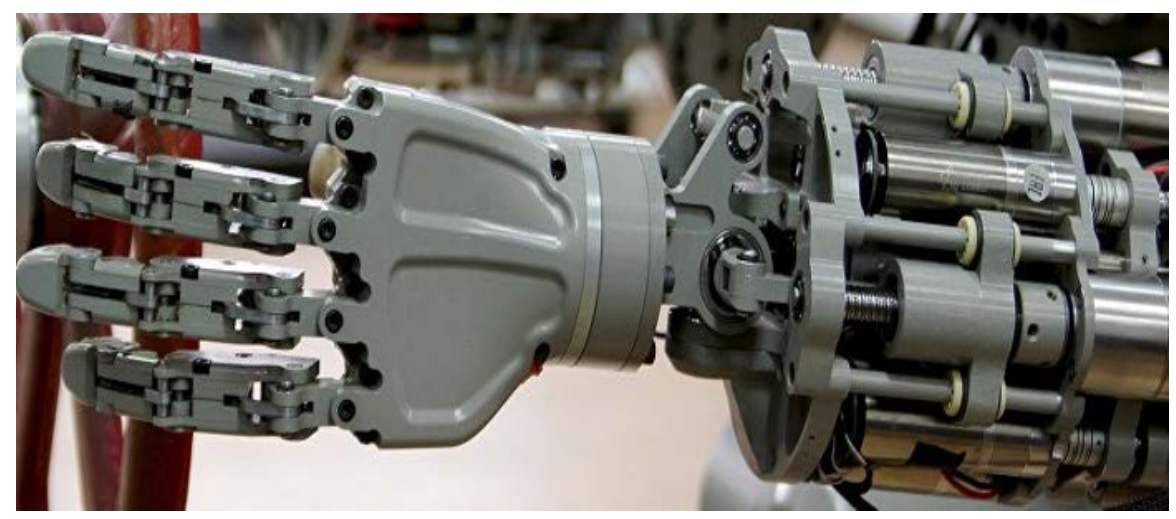

Fig. 1. General view of the AG of the robot "FEDOR"

\section{Statement of a problem}

AR functionality is determined according to its grippers kinematic and power characteristics. In order to provide movements similar to human fingers, relative rotation angles of AGL output links should not be less than: proximal $-90^{\circ}$, medial $-110^{\circ}$, distal $30^{\circ}$. Therein various combinations of realizing rotational angles involving minimum number of motors should be provided. These requirements may be fulfilled due to the realization of new design concepts of motion transfer system (MTS) output links. Previously technical solutions wherein one motor provides the motion in some degrees of freedom without a kinematic dependence $[6,7]$ were defined. MTS construction with the output links common drive, which kinematic scheme is presented in the Figure 2, represents these design concepts development. 


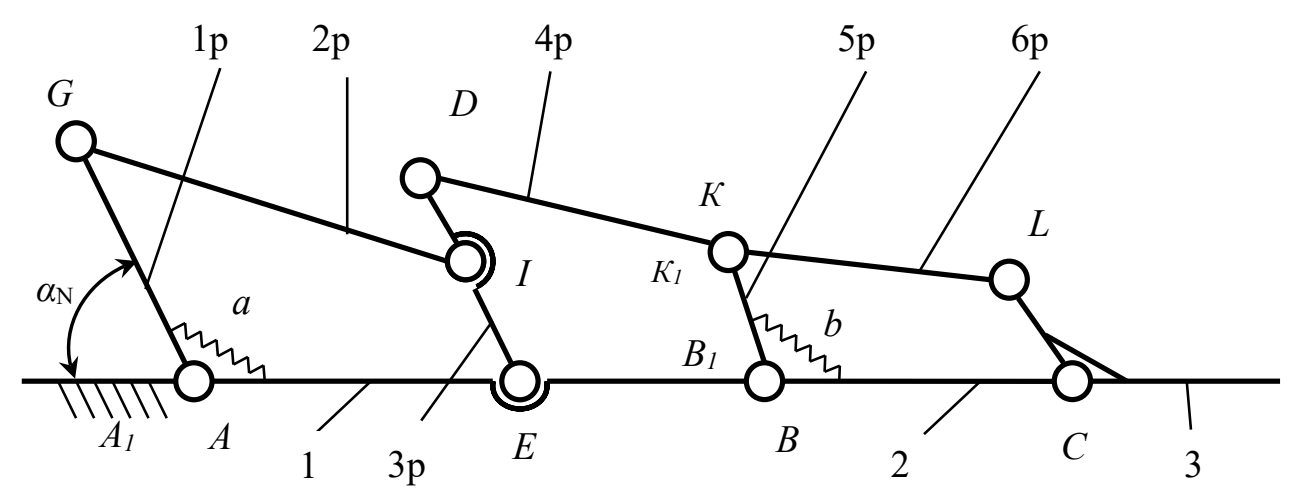

Fig. 2. General AGL kinematic scheme

\section{Functioning of MTS with the Common Drive}

General AGL kinematic scheme consists of a general kinematic scheme comprising output links 1-3, kinematic couples $A, B, C$ and MTS kinematic scheme. Position of the output links $1-3$ is determined by relative rotation angles $\varphi_{1}, \varphi_{2}, \varphi_{3}$. MTS kinematic scheme represents connected double rockers and includes six links $1 \mathrm{p}-6 \mathrm{p}$ and nine kinematic couples $A n, G, D, E, B n, I, K, K_{l}, L$. The drive output link is connected with the link $1 \mathrm{p}$, which initial position is determined by the start angle of the link $1 \mathrm{p}-\alpha_{N}$ adjustment (Fig. $3)$.
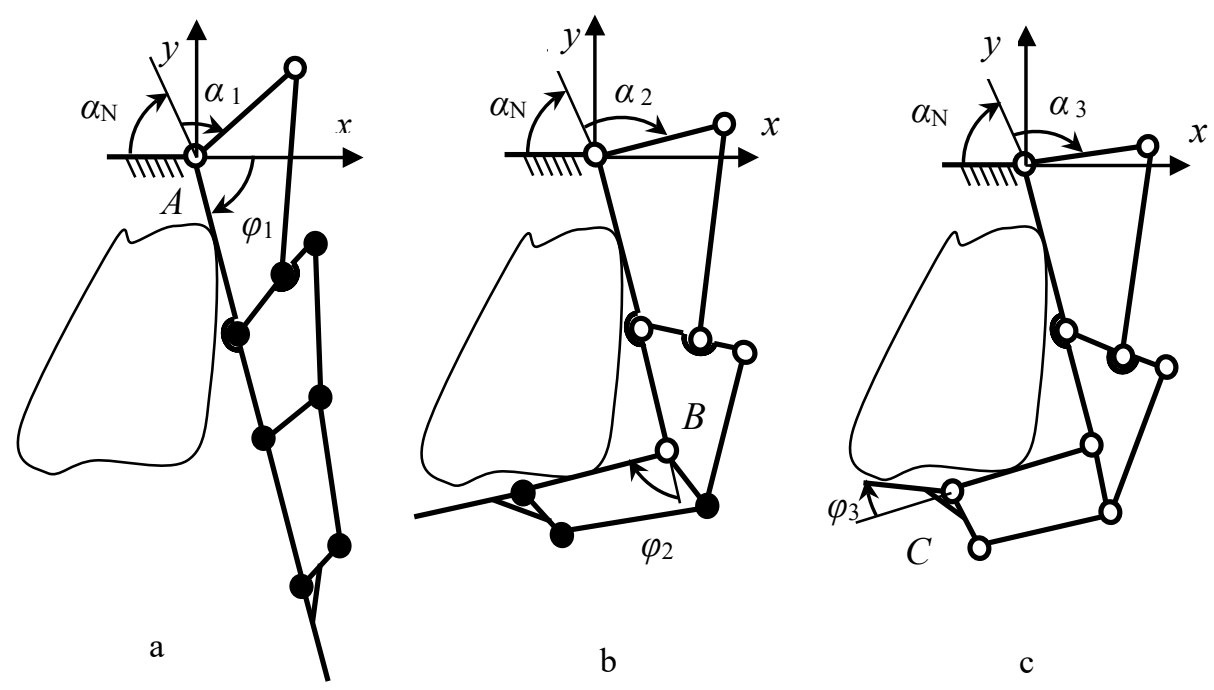

b

C

Fig. 3. Versions (states) of AGL schematic structures depending on interaction with an object surface: $\mathrm{a}-$ first $\varphi_{1}=75^{\circ}, \mathrm{b}-$ second $\varphi_{2}=95^{\circ}, \varphi_{3}=30^{\circ}$. 
AGL is additionally equipped with functioning connections presented as springs: $a, b$. The spring $a$ is mounted between the links 1 and $1 \mathrm{p}$, the spring $b$ - between the links 2 and $5 \mathrm{p}$.

AGL represents a mechanism with the common drive and the variable schematic structure, which is presented in three optional versions (states). Whereby there is only one degree of freedom of the mechanism at any given time, and the output link is the link 1, 2, 3 in sequence. Implementation of the second and the third schematic structure versions is determined by external conditions. In the absence thereof, the first version is applied, where the flexibility takes place only in the couple $A$. Thus, the relative motion of the links 2 and 3 is missing. If the link 1 reaches the surface, it stops (Fig. 3, a), thus the second version of the AGL schematic structure is realized, where the link 2 is an output link, and the motion in the kinematic couple $B$ (Fig. 3, b) is provided. After it reaches the object surface, the third version of the AGL schematic structure with the output link 3 and motion in the kinematic couple $C$ (Fig. 3, c) takes place.

\section{Kinematics of MTS with the Common Drive}

The power analysis is a basis for the development of the AG working draft. For the carrying out of this analysis the determination of links relative position performing at the stage of kinematics researches is required. AGL kinematic scheme (Fig. 1) is distinctly different from known schemes. Therefore, a research method should be defined.

Kinematic analysis refers to determination of the rotational angles of the output links $\varphi_{i}$ in the function of the rotational angle of the driving link $1 p-\alpha$, thus forward kinematics formula. However, resulting from the variability of the AGL schematic structure the definition of the output links relative position corresponding to the schematic structure version, i.e. inverse kinematics formula, should take priority thereof.

The proposed algorithm of the kinematics research considers three stages. At the first stage the rotational angle $\varphi_{1}$ corresponding to reaching a surface (Fig. 3, a) by the link 1 is determined. The calculation takes place, if the link 1 reaches an object surface profile predetermined in axes $x A y$. Whereby the rotational angle of the drive output link $1 \mathrm{p}-\alpha_{1}$ is equal to the rotational angle of the link $\varphi_{1}$.

At the second stage double rockers connected to the output link 1 operate. Implementing formula of the angle $\alpha_{2}=\alpha_{2}\left(\varphi_{1}, \varphi_{2}\right)$ is based on the use of a closed-loop method [8]. Whereby the angle $\varphi_{2}$ as well as at the first stage the angle $\varphi_{1}$ is determined, if the output link 2 reaches the object surface, or is predetermined.

After analytic calculations and changings the dependence $\alpha_{2}=\alpha_{2}\left(\varphi_{1}, \varphi_{2}\right)$ is as follows:

$$
\alpha_{2}=\phi_{1}+\arccos \frac{l_{I G}{ }^{2}+l_{A G}{ }^{2}-t_{2}{ }^{2}}{2 \cdot l_{A G} \cdot t_{2}}+\operatorname{arctg} \frac{-l_{E I} \cdot \sin q}{l_{E I} \cdot \cos q+l_{A E}}-\alpha_{H}
$$

where

$$
\begin{gathered}
t_{2}{ }^{2}=l_{A E}{ }^{2}+l_{E I}{ }^{2}+l_{A E} \cdot l_{E I} \cdot \cos q \\
q=\arccos \frac{l_{D K}{ }^{2}+l_{E D}{ }^{2}-t_{1}^{2}}{2 \cdot l_{E D} \cdot t_{1}}+\operatorname{arctg} \frac{l_{B K} \cdot \sin \phi_{2}}{l_{B K} \cdot \cos \phi_{2}-1}, \\
t_{1}{ }^{2}=l_{E B}{ }^{2}+l_{B K}{ }^{2}-l_{E B} \cdot l_{B K} \cdot \cos \phi_{2} .
\end{gathered}
$$


At the next stage, the third version of the schematic structure is implemented, whereby simultaneous motion of all MTS links provides the movement of the output link 3.

The dependence (in view of mathematical expressions difficulty the latter are not listed here) $\alpha_{3}=\alpha_{3}\left(\varphi_{1}, \varphi_{2}, \varphi_{3}\right)$ is obtained on the basis of the calculations similar to the calculations performed for the second schematic structure.

Rotation of the link $1 \mathrm{p}$ by an angle $134^{\circ}$ provides rotation of AGL output links by angles $\varphi_{1}=90^{\circ}, \varphi_{2}=110^{\circ}, \varphi_{2}=30^{\circ}$ for matching parameters of the kinematic scheme represented in the Figure 1. The required rotational angle of the link $1 \mathrm{p}$ is achieved due to gear and lever mechanisms.

Verification of the presented kinematic analysis method is carried out with the use of graphical models.

\section{Conclusion}

Given kinematic scheme of MTS with a common drive provides consequent motion of output links with rotational angles aligned with human fingers movements.

Obtained analytical dependences of the rotational angle of the drive output link allow to determine relative position of AGL link sufficient for the power analysis in the function of the rotational angles of the output links $\alpha_{i}=\alpha_{i}\left(\varphi_{i}\right)$.

Functional dependence of the rotational angle $\alpha$ allows to estimate the impact of MTS links lengths and initial setting on AGL links position upon contact with a capturing object.

\section{References}

1. I.G. Zhidenko, A.A. Bogdanov, I.M. Kutlubayev, V.B. Sychkov, Reshetnev's Readings: Proc. of the 17th Int. Scientific Conf. Dedicated to the Member of the Academy M.F. Reshetnev (November, 12 - 14, 2013, Krasnoyarsk). Krasnoyarsk: Siberian State Aerospace University, Basis of structural scheme selection of space application robots, 1, 278-80 (2013)

2. J. Badger, D. Gooding, K. Ensley, K. Hambuchen, A. Thackston, ROS in Space. The Complete Reference, A Case Study on Robonaut 2, 1, 343-73 Doi: https://doi.org/10.1007/978-3-319-26054-9

3. V.G. Sorokin, Manned Spaceflight, 1 (22), 64-68 (2017)

4. S.A. Katchanov, V.B. Moshkov, A.Y. Barannik, A.V. Yakutov, Civil Security Technology, Adapting anthropomorphic robotic system technologies to solve the tasks of EMERCOM Russia, 13. No. 4 (50) 14-8 (2016)

5. A.A. Bogdanov, M.R. Iksanov, I.M. Kutlubayev, Poleznaya model zakhvata (Utility Model of the Gripper), Patent RF, no. 173229 (2017)

6. A.A. Bogdanov, A.S. Gorbanyova, I.M. Kutlubaev, Yu.I. Kutlubayeva, Proc. of the 18th Int. Scientific Conf. Dedicated to the Member of the Academy M.F. Reshetnev (September, 10-14, 2014, Krasnoyarsk): 2 parts, ed. by Y.Y. Loginova. Krasnoyarsk: Siberian State Aerospace University, Principles of anthropomorphic capture constructions with function element group drive, 1, 273-4 (2014)

7. A.A. Bogdanov, I.G. Zhidenko, Yu.I. Kutlubayeva, A.F. Permyakov, Patent RF na izobreteniye Zakhvat (Patent RF on utility invention Gripper), no. 2570597 (2015)

8. I.I. Artbolevskij, Mechanism and Machine Theory, Moscow: Nauka (Teoriya mekhanizmov i maschin) 638 (1975) 